\section{Clintad âdecoros} OF THE LOCK HOSPITAL. BY

H E N R Y L E E, EsQ., F.R.C.S., SURGEON TO THE hOSPITAI.

\section{I.-A Chinical Lecture.}

IF there be one pathological fact that has been more frequently reiterated of late years than another, it is, that a primary syphilitic sore may be distinguished from every other affection by its secretion, when inoculated, invariably producing the characteristic syphilitic pustule. Although syphilitic inoculation was practised by Hunter and other accurate observers, it remained for Ricord to draw welldigested conclusions from the facts which this mode of investigation presented for our interpretation. By patient experiment and rigorous reasoning, Ricord established the truth, which has now been confirmed throughout Europe from Italy to Norway; namely, that certain forms of syphilitic sores will produce, when inoculated, their like, which in their turn will again furnish a secretion capable of being again inoculated with similar results. It would be difficult to overrate the importance of this discovery, so analogous to that of the small-pox inoculation. But practical men have, nevertheless, been unable to see in the experiments in question a conclusive representation of that which they daily observe in practice. That certain forms of primary syphilitic disease will produce the characteristic pustule, when inoculated, all must allow; but it does not follow, on the one hand, that all primary syphilitic sores are alike capable of being inoculated; nor, on the other, that when inoculated, they will necessarily yield the same result. Under these circumstances, and without proof as to these two last mentioned particulars, to say that a sore is syphilitic because it produces a characteristic pustule when inoculated, and that it produces a characteristic pustule because it is syphilitic, is manifestly to reason in a vicious circle. The propositions themselves are undoubtedly true; but they cannot, without further proof, be received as excluding other modes of syphilitic infection and other forms of primary syphilis.

We are led then to ask, in the first place, whether there is no form of primary syphilis except that which commences as a pustule. This question is important, since surgeons engaged in practice very seldom have an opportunity of observing the primary syphilitic pustule; and still less frequently have they an opportunity of tracing any secondary symptoms consequent upon it. Now, during the years 1855 and 1856, we examined at this hospital the secretion from a large number of primary syphilitic sores, and amongst these were ninety-five cases presenting all the ordinary characters of the primary infecting sores. The secretions from these ninetyfive cases consisted of epithelial débris floating in a serous fluid, or of globules of various shapes and sizes, which did not, upon the addition of acetic acid, yield the well defined nuclei characteristic of pus. In a very considerable number of cases, the secretion appeared to consist of nothing but epithelial débris and serous fluid, and in some the sores yielded no fluid at all.

What reason, then, have we to believe that these primary sores commenced as pustules ; or that, unless artificially irritated, they would produce pus at all? And, secondly, what evidence have we that every syphilitic sore produces a secretion capable of being inoculated in the same manner? It is to this point that I wish principally to devote this lecture.

When the secretion is taken from a chancre during its period of progress, or when stationary, and properly inoculated, the following results may, according to M. Ricord, be invariably observed. During the first twenty-four hours, the inoculated point becomes red; from the fourth to the fifth day, the secretion becomes purulent; and a pustule, with a depression in its centre resembling those of smallpox, is fully formed. (Traité Pratique, p. 89.) The inoculation never fails when properly performed. "L'inoculation n'échoue jamais quand on prend le pus dans les conditions voulues et qu'on l'applique bien" (p. 94). The appearance produced is said to be regular, characteristic, and uniform (p. 135). It is useless to multiply quotations upon this point, the facts concerning which have been so generally received and so often republished.

In the year 1856, I published an account of some cases in which, notwithstanding the assertions so unreservedly made and so generally received, I had not been able to produce any effect by ordinary inoculation. The experiments were tried in this hospital, care being taken to select instances in which the sores had not begun to heal; and among the cases were the following.

Thomas C., aged 16, was admitted on the 2rth of November, with an indurated sore extending half-way round the margin of the prepuce, causing phimosis. This had commenced a fortnight previously. The secretion from the sore was carefully inoculated on the patient's thigh in several points. The inoculations were followed by no result, and the patient was soon afterwards affected with secondary syphilis.

James G., aged 25, had a large indurated sore near the orifice of the prepuce, which had appeared as a pimple four weeks previously. The secretion was carefully inoculated in several points, but without result. Constitutional syphilis followed.

Matilda P. presented on the left external labium a well marked indurated sore, which she stated had existed one week only. The secretion, which consisted of a thin serous fluid, was carefully inoculated. No result followed the inoculation.

Julia $B$, aged 21, had a red glazed sore on the external labium, surrounded by distinct specific induration. The disease had commenced three weeks previously, as a pimple. The secretion from the surface of the sore was inoculated without result.

Since the period above referred to, we have inoculated the secretion from a considerable number of sores presenting the characters of the specific adhesive inflammation; and, as a rule, no result has been obtained where no artificial irritation has been applied. The secretion in these cases has consisted chiefly of epithelial débris floating in serum more or less turbid. The character of the secretion of these sores may readily be altered by anything that is brought in contact with them. The application of caustic, or a thick scab which confines the secretion, 
or a piece of linen which sticks to the sore, will produce a temporary discharge more or less puriform ; but dress the part with wet lint for a day or two, and the natural character of the discharge will again be evident.

As a rule, then, I say (at least, that has been our experience at this hospital) that this kind of primary sore is not, under ordinary circumstances, capable of being inoculated with the lancet; and we therefore cannot but come to the conclusion that those who have maintained that all primary syphilitic sores can be alike inoculated have generalised too hastily. But a much more important question remains; namely, Does it follow that, because these sores are not capable of being inoculated by the lancet in their ordinary condition, therefore they cannot be communicated at all ? And, if capable of being inoculated under certain circumstances, are the results produced identical with those produced by the inoculation of the secretion from suppurating sores?

In order to determine these questions, I would request your particular attention to some cases now in the hospital, and particularly to that of a boy named $A$. The details of this case, and the results of the inoculations performed, I will immediately mention; but, before doing so, I would draw your attention to the fact that in practice we continually meet with cases which in their early stages are similar to the one I am about to describe, and which, with or without treatment, become covered by epithelium; and, although the specific induration remains, yet the surface of these sores affords no fluid secretion whatever. Inoculation in the ordinary way at this period of the disease would be entirely out of the question. But, if a person in the condition I have mentioned get married, his wife will have a good chance, even before her pregnancy, of becoming infected with syphilis. Some very distinct cases where this has happened have fallen under my own observation. How, then, is the disease communicated in these instances? We have here a rather numerous class of cases in which the sores have become covered over with cuticle, which yield no pus nor fluid secretion of any kind, and yet which are capable of communicating infection from one individual to another. How, I ask, does the inoculation take place from a sore affected only with the specific adhesive inflammation either before or after that sore has apparently healed? Some light will, I think, be thrown upon these questions by the careful consideration of the facts presented to our notice in the boy A., at present in the hospital.

This lad had had gonorrhœa six months before his present attack, but otherwise he had never had any venereal symptoms. His present disease was of about a fortnight's duration. He first perceived a superficial sore behind the corona glandis, which healed in a few days. Two or three days after the first appearance of this sore, a little pimple appeared on the outer skin of the prepuce. This was squeezed, and discharged a watery fluid. A circular sore then formed, which continued to increase. He applied to me on the 25th of July, with a well marked Hunterian chancre. This was of a circular form, surrounded by well marked and accurately defined induration, and discharged a white turbid secretion. This fluid was placed under the microscope, having previously been mixed with a little acetic acid. It contained no pus-globules. The glands in the groin were enlarged and indurated, but not inflamed.
July 27th. The secretion from the sore was inoculated in several points on the patient's thigh.

July 29th. The boy was admitted into the Lock Hospital. The inoculations had been followed by no result. The secretion from the sore was again examined, and found to contain no pus.

July 31st. The sore, which continued to increase in size, had been dressed with linen and cold water since last report. The linen appeared to have irritated the sore in some degree, and numerous globules now appeared in the secretion; but, upon the addition of acetic acid, the distinct outline of the pus-nuclei ${ }^{\circ}$ were not visible. Several fresh inoculations were made. The sore was dressed with wet lint.

Aug. 3rd. None of the inoculations had succeeded. The secretion from the surface of the sore, placed under the microscope and treated with acetic acid, did not appear to contain any pus. The sore was now ordered to be dressed with the acetum lyttæ. The glands at the back of the neck were now enlarged, and skin presented for the first time the appearance of a syphilitic eruption.

Aug. 5th. None of the former inoculations had been followed by any effect. The application of the acetum lyttæ had produced a superficial slough on the surface of the sore, and blistered the surrounding skin. Some distinct pus-globules were now visible in the secretion from beneath the slough. This secretion was inoculated upon the thigh in several points.

Aug. 7th. The sore now again secreted no pus. Fresh inoculations were performed.

Aug. 10th. The sore was dressed twice yesterday with the unguentum sabinæ, and it now yielded a copious secretion of pus, This was inoculated in several points in a fresh place upon the thigh.

Aug. 12th. The inoculations last made had succeeded. The primary sore still yielded a copious secretion of pus.

Aug. 14th. The sore had been dressed with lint, kept wet with water since last report, and now no pus could be detected in the secretion. The inoculations both of the 5th and of the 10th had now succeeded. They presented the appearance of circular red patches, with some elevation and thickening of the cuticle. In one place there was the appearance of a broken vesicle, from which a serous secretion exuded. This secretion from the inoculation was again inoculated on the thigh.

Aug. 17th. The inoculation from the inoculation had succeeded. It presented the appearance of a red circular patch, from which the cuticle was abraded, with slight thickening of the skin. It had not at all assumed the appearance of a pustule, nor was anything like pus secreted from its surface. A single pustule, surrounded with very little inflammation, had formed in one of the points first inoculated. The eruption on the surface of the skin was fading. All the inoculations in this case were made with a lancet used for no other purpose, kept carefully wiped and wrapped in paper.

Aug. 19th. The inoculations appeared as separate red patches on the skin. The skin in these situations was slightly raised and thickened, but no induration extended into its substance. The solitary pustule which had appeared had dried up. The original sore was healing; but, being dressed with the unguentum sabinæ, it afforded a purulent secretion, which was inoculated upon another patient who was apparently suffering from organic disease of the liver.

Aug. 21st. The inoculations presented the same appearances as before; they appeared covered in certain parts with thin scales. They were tender to the touch, with slight thickening on the surface, which did not extend into the substance of the cutis. The inoculations nowhere presented any appearance of ulceration. The inoculations performed on the 19th had produced a small dark brown circular patch, in which the skin was slightly 
elevated. The secretion from the original sore was now again inoculated on a second female already affected with constitutional syphilis.

Aug. 24th. One of the inoculations on the boy had a slight tendency to ulcerate; the others were desquamating and losing their colour.

The single inoculation on the first woman had assumed the form of a small red pimple. The redness gradually faded into the colour of the surrounding skin. The cuticle at the inoculated part was thickened and elevated.

The inoculation on the second woman, already affected with constitutional syphilis, was less marked. There was only a slight redness and elevation at the inoculated part.

The particulars of these last two cases we may follow out on a future occasion. From the facts which have already been brought under notice, the following very important points are proved.

1. That some primary syphilitic sores cannot readily be inoculated in the ordinary way upon the patient who has them, either during the period of progress or afterwards.

2. That the sores which are not capable of being thus inoculated, as far as we have hitherto seen, are those affected with specific adhesive inflammation, and which do not, except under conditions of artificial irritation, secrete pus.

3. That these sores, although not capable of being inoculated in the ordinary way, will nevertheless, when irritated, furnish a secretion which is capable of being inoculated apon a patient already affected with syphilis.

4. That the inoculations thus produced do not give rise, as a rule, to either suppuration or ulceration, but to some adhesive form of inflammation.

The inoculations to which attention has now been directed, and the results of which may be seen in $t$ wo other patients now in the hospital besides those to whomI have above referred, have all assumed a remarkably uniform character. There are many points of the greatest interest connected with this newly demonstrated form of artificial inoculation, which must be carefully distinguished from the effects of inoculation upon a patient not previously diseased. Here, after a certain period of incubation, a well marked Hunterian chancre would probably have been the result of each inoculation performed for the first time.

Dr. Wrnship Outdone. Dr. Winship, the celebrated Massachusetts athlete, who was asserted to be the "strongest man in the world," has met a superior in the person of one William Thompson, who is connected with the Chicago Gymnasium. The test of strength oc. curred in that city one day, at a gymnastic tournament, at which Dr. Winship performed his great muscular feat of lifting nine kegs of nails, weighing 1000 pounds, and raising, with the aid of harness on his shoulders, 1517 pounds. He was succeeded by Thompson, who, commencing with the last lift of the Doctor, then went on adding weights, and lifting with harness on shoulders and hips, until the numbers stood successively, 1536, $1636,1736,1836,1936,2036,2136$ pounds-a very remarkable lift the latter, to be sure. $\mathrm{He}$ also experimented with dumb-bells weighing 100 and 165 pounds. Another competing gymnastic, named Curtis, "pushed" first 130 pounds, and then 150 pounds in each hand with the pulley, and, lying down upon his back, put up 110 pounds in each hand. (American Paper.)

\section{Goriginal Communications.}

\section{THE GALVANO-CAUSTIC APPARATUS: ITS APPLICATIONS IN SURGERY.}

By Thomas James Walker, M.B.Lond., late AssistantPhysician and Pathologist to the Queen's Hospital, and Demonstrator of Anatomy in Queen's College, Birmingham.

THE galvano-caustic apparatus is extensively used in Vienna for the removal of morbid growths, as well as for other purposes. Owing to the absence, during the greater part of my stay last year in the Austrian metropolis, of Dr. Adolph Zsigmondy, Primararzt in the Hospital, who is the great advocate of the galvano-caustic mode of operating, I saw less of it than I should otherwise have done.

In the skin wards for the treatment of lupus, I several times witnessed its employment as a caustic by Professor Hebra. To all the parts affected, the instrument was applied at a white heat; thus, in a case of lupas of the ear, the porcelain cauteriser was deliberately applied to the whole surface of that organ. During the operation, which was necessarily a slow one, the size of the instrument being about that of an ordinary lead-pencil, the patient, a nervous female, continued shrieking violently. In another case, almost cured, the small pointed platinum cauteriser was applied to the numerous points, where new centres of the inveterate inflammation were appearing upon the healed parts. During this process, the patient, a boy, scarcely uttered a groan; but it is hard to say whether this depended on absence of suffering, or on the fortitude produced by the prospect of one or two ten-kreutzer pieces which the professor gave him for his good behaviour, when the operation was completed. Similar courage, or freedom from pain, was manifested by a young man in another case, where the cautery was applied round the edges of the several large patches of lupus non exedens, which existed about his body.

In Dr. Zsigmondy's practice, I had an opportunity of seeing the employment of this instrument, as a cautery to various ulcers of the leg. The galvano-caustic instrument in these cases, where the surface to be treated was large, did not avail for the completion of the operation; the aid of the ordinary actual cautery being called in, on account of the much larger heated surface which the iron heated in a charcoal fire affords, as compared with the small portion of platinum or porcelain which can be heated by the galvanic current. The first application of the glowing cautery to these parts appeared exquisitely painful, there being of course no particular virtue in the heat produced by electricity which should prevent its causing pain; but, in comparing the actual cautery as usually heated with that heated by the continuous voltaic current, as regards the pain caused by their application, we find that the latter, possessing in itself the source of heat, is not cooled by the tissues of the body, consequently it quickly and completely destroys their sensibility and vitality, producing only a momentary pain; while the former, however massive it may be made in order that it may retain its heat, is, on its first application to the tissues, so cooled by them, that it requires a longer application in order completely to destroy the structures with which it is in contact. and may in many places only half burn them, causing therefore pain of some duration. This I would advance as a general rule. To the eschar left where the instrument had been once applied in these cases of ulcer, it might immediately afterwards be reapplied without producing 